\title{
Hausdorff Dimension and Quadratic Relations between Frequencies of Digits
}

\section{Luis Barreira}

Departamento de Matemática, Instituto Superior Técnico, 1049-001 Lisboa, Portugal

E-mail address: barreira@math.ist.utl.pt

URL: http://www.math.ist.utl.pt/ barreira

\section{Claudia Valls}

Departamento de Matemática, Instituto Superior Técnico, 1049-001 Lisboa, Portugal

E-mail address: cvalls@math.ist.utl.pt

\begin{abstract}
We describe how the multifractal analysis of dynamical systems can be used to compute the Hausdorff dimension of a large class of sets in the real line, defined in terms of relations between the frequencies of digits in some integer base. As an illustration we compute the Hausdorff dimension of a class of sets in $[0,1]$ that are defined in terms of linear or quadratic relations between the frequencies of digits. Although these relations are only prototypes of more general ones, the computations follow the same procedure and are already rather involved.
\end{abstract}

\section{Introduction}

Given an integer $m>1$, for each number $x \in[0,1]$ we denote by $0 . x_{1} x_{2} \cdots$ a base- $m$ representation of $x$. It is easy to see that the representation is unique except for countably many points. For each $k \in$ $\{0, \ldots, m-1\}, x \in[0,1]$, and $n \in \mathbb{N}$ we set

$$
\tau_{k}(x, n)=\operatorname{card}\left\{i \in\{1, \ldots, n\}: x_{i}=k\right\},
$$

Supported by the Center for Mathematical Analysis, Geometry, and Dynamical Systems, and through Fundação para a Ciência e a Tecnologia by Program POCTI/FEDER, Program POSI, and the grant SFRH/BPD/26465/2006.

Key words: frequencies of digits, Hausdorff dimension. 
and

$$
\tau_{k}(x)=\lim _{n \rightarrow \infty} \frac{\tau_{k}(x, n)}{n}
$$

whenever the limit exists. The number $\tau_{k}(x)$ is called the frequency of the digit $k$ in the base- $m$ representation of $x$. Consider the set

$$
F_{m}\left(\alpha_{0}, \ldots, \alpha_{m-1}\right)=\left\{x \in[0,1]: \tau_{k}(x)=\alpha_{k} \text { for } k=0, \ldots, m-1\right\},
$$

where $\alpha_{k} \in[0,1]$ for each $k$, and $\sum_{k=0}^{m-1} \alpha_{k}=1$. Eggleston showed in [5] that this set has Hausdorff dimension

$$
\operatorname{dim}_{H} F_{m}\left(\alpha_{0}, \ldots, \alpha_{m-1}\right)=-\sum_{k=0}^{m-1} \alpha_{k} \log _{m} \alpha_{k}
$$

(with the convention that $0 \log 0=0$ ). A related result was first obtained by Besicovitch in [3] when $m=2$. It follows immediately from (1) that if $\alpha_{k} \in(0,1)$ for some $k$, then $\operatorname{dim}_{H} F_{m}\left(\alpha_{0}, \ldots, \alpha_{m-1}\right)>0$, and thus the set $F_{m}\left(\alpha_{0}, \ldots, \alpha_{m-1}\right)$ is nonempty and actually dense in $[0,1]$. The work of Eggleston was further generalized by Billingsley with a more unified approach (see his book [4] for details and references). See also [2, 1] for the discussion of more recent developments and for further references.

Our main aim is to illustrate how the theory of multifractal analysis of dynamical systems can be used to compute the Hausdorff dimension of a large class of sets in the real line. For definiteness, we consider sets in $[0,1]$ that are defined in terms of general linear or quadratic relations between two frequencies of digits (we note that in the case of the set $F_{m}\left(\alpha_{0}, \ldots, \alpha_{m-1}\right)$ there are no relations between the frequencies). More precisely, we consider a quadratic polynomial

$$
Q(x, y)=a x^{2}+b x y+c y^{2}+d x+e y+f,
$$

and given $m>2$ we define the set

$$
F=\left\{x \in[0,1]: Q\left(\tau_{0}(x), \tau_{1}(x)\right)=0\right\} .
$$

It follows from a general approach based on the multifractal analysis of dynamical systems, as described in [2], that the Hausdorff dimension of $F$ is given by

$$
\operatorname{dim}_{H} F=-\min \left\{\sum_{k=0}^{m-1} \alpha_{k} \log _{m} \alpha_{k}:\left(\alpha_{0}, \ldots, \alpha_{m-1}\right) \in G\right\},
$$

where

$$
G=\left\{\left(\alpha_{0}, \ldots, \alpha_{m-1}\right) \in[0,1]^{m}: Q\left(\alpha_{0}, \alpha_{1}\right)=0, \sum_{k=0}^{m-1} \alpha_{k}=1\right\} .
$$


For simplicity of the exposition we always assume that $b=0$, thus with the polynomial $Q$ already written in "normal form". We note that the computations are already rather involved in this case. We consider separately the linear case and the quadratic case, respectively in Sections 2 and 3. See also the beginning of Section 3 for a related "geometric" discussion when $b \neq 0$.

\section{The linear case}

2.1. General formulas. When $a=c=0$ and $(d, e) \neq(0,0)$ the relation $Q(x, y)=0$ describes a straight line. More concretely, $Q(x, y)=d x+e y+f$ and thus,

$$
\operatorname{dim}_{H} F=-\min \left\{\sum_{k=0}^{m-1} \alpha_{k} \log _{m} \alpha_{k}: d \alpha_{0}+e \alpha_{1}+f=0, \sum_{k=0}^{m-1} \alpha_{k}=1\right\} .
$$

We have the following result.

Theorem 1. Assume that $a=c=0$ and $(d, e) \neq(0,0)$. When $F \neq \varnothing$ we have:

1. if de $=0$ then setting $\kappa=\max \{d, e\}$,

$$
\operatorname{dim}_{H} F=\frac{f}{\kappa} \log _{m} \frac{-f}{\kappa}-\frac{\kappa+f}{\kappa} \log _{m} \frac{\kappa+f}{\kappa(m-1)} ;
$$

2. if $d e \neq 0$ then

$$
\operatorname{dim}_{H} F=-\min \left\{K\left(\alpha_{0}\right): \alpha_{0},-A-B \alpha_{0} \in[0,1]\right\},
$$

where $A=f / e, B=d / e$, and

$$
\begin{aligned}
K(\alpha)= & \alpha \log _{m} \alpha+(-A-B \alpha) \log _{m}(-A-B \alpha) \\
& +(1-\alpha+A+B \alpha) \log _{m} \frac{1-\alpha+A+B \alpha}{m-2} .
\end{aligned}
$$

Proof. Case $d \neq 0, e=0$. Since $F \neq \varnothing$ we must have $\alpha_{0}=-f / d \in[0,1]$, and thus, by (3) we obtain

$$
\operatorname{dim}_{H} F=\frac{f}{d} \log _{m} \frac{-f}{d}-\min \sum_{k=1}^{m-1} \alpha_{k} \log _{m} \alpha_{k},
$$

with the minimum taken over all $\alpha_{1}, \ldots, \alpha_{m-1} \in[0,1]$ such that $\sum_{k=1}^{m-1} \alpha_{k}=$ $1-\alpha_{0}$. Since the function $x \mapsto-x \log x$ is strictly convex, the minimum is attained only when $\alpha_{1}=\cdots=\alpha_{m-1}=\left(1-\alpha_{0}\right) /(m-1)$, and thus

$$
\operatorname{dim}_{H} F=\frac{f}{d} \log _{m} \frac{-f}{d}-\frac{d+f}{d} \log _{m} \frac{d+f}{d(m-1)} .
$$


Case $d=0$ and $e \neq 0$. Proceeding as above, since $F \neq \varnothing$ we must have $\alpha_{1}=-f / e \in[0,1]$, and thus,

$$
\operatorname{dim}_{H} F=\frac{f}{e} \log _{m} \frac{-f}{e}-\frac{e+f}{e} \log _{m} \frac{e+f}{e(m-1)} .
$$

Case de $\neq 0$. Note that

$$
\alpha_{1}=-A-B \alpha_{0} .
$$

Using again the strict convexity of the function $x \mapsto-x \log x$, it follows from (3) that $\operatorname{dim}_{H} F=-\min K\left(\alpha_{0}\right)$, with the minimum taken over all $\alpha_{0} \in[0,1]$ such that $\alpha_{0}+\alpha_{1} \in[0,1]$. The desired result follows from (6).

To determine the minimum in (4) it helps to consider the derivative

$$
K^{\prime}(\alpha)=\log _{m} \alpha-B \log _{m}(-A-B \alpha)+(B-1) \log _{m} \frac{1+A+(B-1) \alpha}{m-2} .
$$

The equation $K^{\prime}\left(\alpha_{0}\right)=0$ is thus equivalent to

$$
\alpha_{0}\left(\frac{1+A+(B-1) \alpha_{0}}{m-2}\right)^{B-1}=\left(-A-B \alpha_{0}\right)^{B} .
$$

Furthermore

$$
K^{\prime \prime}\left(\alpha_{0}\right) \log m=\frac{1}{\alpha_{0}}+\frac{(B-1)^{2}}{1+A+(B-1) \alpha_{0}}+\frac{B^{2}}{-A-B \alpha_{0}},
$$

and $K^{\prime \prime}\left(\alpha_{0}\right)>0$ when the three denominators in $(8)$ are in $(0,1)$, in which case any solution $\alpha_{0}$ of the equation $K^{\prime}\left(\alpha_{0}\right)=0$ is in fact the unique solution. The remaining cases (corresponding to the boundary of $[0,1]$ ) can be treated by direct substitution in (5) (for example, if $A=0$, i.e., $f=0$, then $\alpha_{0}=0$ and thus $\left.\operatorname{dim}_{H} F=\log (m-2) / \log m\right)$.

2.2. Examples. We now consider several values of $B=d / e$ in order to illustrate the variety of formulas for the Hausdorff $\operatorname{dimension} \operatorname{dim}_{H} F$ when $d e \neq 0$. This essentially amounts to solve equation (7). For example, when $B \in \mathbb{Q}$ the problem is reduced to solve an algebraic equation. More precisely, writing $B=p / q$ with $p \in \mathbb{Z}, q \in \mathbb{N}$ and $(p, q)=1$, the degree $D$ of this equation is given by

$$
D= \begin{cases}p & \text { if } p>0 \text { and } B \geq 1 \\ q+1 & \text { if } p>0 \text { and } B<1 \\ |p|+q & \text { if } p<0\end{cases}
$$


Example 1. Let $B=1$. Then (7) yields $\alpha_{0}=-A / 2$. By (6) we must have $-A=\alpha_{0}+\alpha_{1} \in[0,1]$ so that $F \neq \varnothing$. Therefore, $\alpha_{0}=\alpha_{1}=-A / 2$ and

$$
\operatorname{dim}_{H} F=A \log _{m} \frac{-A}{2}-(1+A) \log _{m} \frac{1+A}{m-2} .
$$

We now consider three examples that lead to quadratic equations.

Example 2. Let $B=2$. Then (7) becomes

$$
\frac{\alpha_{0}\left(1+A+\alpha_{0}\right)}{(m-2)\left(A+2 \alpha_{0}\right)^{2}}=1 \text {. }
$$

Setting

$$
\lambda=\sqrt{1+A(2+A)(9-4 m)},
$$

it follows from (9) that

$$
\alpha_{0}=\frac{A(9-4 m)+1 \pm \lambda}{8 m-18} \text { and } \quad \alpha_{1}=-\frac{1 \pm \lambda}{4 m-9} .
$$

Since $4 m-9>0$, we must take the minus sign in (11). Furthermore, we must have $\lambda \geq 1$, and thus, by (10), $-2 \leq A \leq 0$. To verify that $\alpha_{0} \geq 0$, we note that

$$
\lambda \leq \sqrt{1+2 A(9-4 m)+A^{2}(9-4 m)^{2}}=1+A(9-4 m) .
$$

Furthermore, since $A \in[-2,0]$ we can verify that $\alpha_{0}+\alpha_{1} \leq 1$. Therefore,

$$
\operatorname{dim}_{H} F=-K\left(-\frac{A}{2}+\frac{1-\lambda}{8 m-18}\right) .
$$

Example 3. Let $B=-1$. In this case (7) becomes

$$
(m-2)^{2} \alpha_{0}\left(\alpha_{0}-A\right)=\left(1+A-2 \alpha_{0}\right)^{2} .
$$

When $m=4$ we obtain

$$
\alpha_{0}=\frac{1}{4}(1+A)^{2} \geq 0 \quad \text { and } \quad \alpha_{1}=\frac{1}{4}(1-A)^{2} \geq 0 .
$$

So that $F \neq \varnothing$ we must have $\alpha_{0}+\alpha_{1}=\left(A^{2}+1\right) / 2 \leq 1$, and thus $A \in[-1,1]$. In this case

$$
\begin{gathered}
\operatorname{dim}_{H} F=1-\frac{(1-A)^{2}}{4} \log _{2}(1-A)-\frac{(1+A)^{2}}{4} \log _{2}(1+A) \\
-\frac{1-A^{2}}{8} \log _{2} \frac{1-A^{2}}{4} . \\
\text { If } m \geq 5 \text { then } \lambda=\sqrt{A^{2} m(m-4)+4} \text { is real, and } \\
\alpha_{0}=\frac{-4+A m(m-4) \pm(m-2) \lambda}{2 m(m-4)} .
\end{gathered}
$$


So that $\alpha_{0} \geq 0$ we must take the plus sign in (12). Therefore,

$$
\alpha_{1}=\frac{-4-A m(m-4)+(m-2) \lambda}{2 m(m-4)}, \quad \alpha_{0}+\alpha_{1}=\frac{-4+(m-2) \lambda}{m(m-4)} .
$$

In order that $\alpha_{0}+\alpha_{1} \leq 1$ we must have $A \in[-1,1]$, in which case

$$
\operatorname{dim}_{H} F=-K\left(\frac{-4+A m(m-4)+(m-2) \lambda}{2 m(m-4)}\right) .
$$

One can show that (13) also holds when $m=3$.

Example 4. Let $B=1 / 2$. Then (7) becomes

$$
2 \alpha_{0}(m-2)^{1 / 2}=\left(-2 A-\alpha_{0}\right)^{1 / 2}\left(2+2 A-\alpha_{0}\right)^{1 / 2} .
$$

Since $\alpha_{0}<-2 A$ (or the second term in (5) wouldn't be defined), we have

$$
\alpha_{0}=-\frac{1 \pm \lambda}{4 m-9}, \quad \lambda=\sqrt{1-4 A(1+A)(4 m-9)} .
$$

So that $\alpha_{0} \geq 0$ we must take the plus sign in (14). Thus, we must have $\lambda \leq 1$ and $A \in \mathbb{R} \backslash(-1,0)$. Moreover, so that $\alpha_{0}<-2 A$ we must have $A>-1 /(2(4 m-9))$. Furthermore,

$$
A \in\left(-\frac{1}{2}-\frac{\sqrt{18-17 m+4 m^{2}}}{4 m-9},-\frac{1}{2}+\frac{\sqrt{18-17 m+4 m^{2}}}{4 m-9}\right),
$$

so that $\lambda \in \mathbb{R}$, and in order that

$$
\alpha_{1}=\frac{-2 A(4 m-9)+1-\lambda}{4 m-9} \geq 0
$$

we must have $A<1 /(2(4 m-9))$. This last value is easily shown to be larger than the right endpoint in (15). Summarizing, we must have

$$
A \in\left(0,-\frac{1}{2}+\frac{\sqrt{18-17 m+4 m^{2}}}{4 m-9}\right) \text {. }
$$

For these values of $A$ it is easy to check that

$$
\alpha_{0}+\alpha_{1}=\frac{-2 A(4 m-9)-1+\lambda}{2(4 m-9)} \leq 1 .
$$

Therefore, $A$ satisfies (16) if and only if $F \neq \varnothing$, in which case

$$
\operatorname{dim}_{H} F=-K\left(\frac{-1+\lambda}{4 m-9}\right) .
$$

We finally present an example that leads to a cubic equation. 
Example 5. Let $B=-2$ and $A=0$. In this case (7) becomes

$$
\left(-5+48 m-24 m^{2}+4 m^{3}\right) \alpha_{0}^{3}-27 \alpha_{0}^{2}+9 \alpha_{0}-1=0 .
$$

Since $m>2$ we have $-5+48 m-24 m^{2}+4 m^{3}>0$, and thus,

$$
\alpha_{0}^{3}+a_{1} \alpha_{0}^{2}+b_{1} \alpha_{0}+c_{1}=0
$$

where

$$
a_{1}=-\frac{27}{a}, \quad b_{1}=\frac{9}{a}, \quad c_{1}=-\frac{1}{a}, \quad a=-5+48 m-24 m^{2}+4 m^{3} .
$$

Set

$$
\begin{aligned}
& P=\frac{3 b_{1}-a_{1}^{2}}{3}=\frac{36(m-2)^{3}}{a^{2}}, \\
& Q=\frac{2 a_{1}^{3}}{27}-\frac{a_{1} b_{1}}{3}+c_{1}=-\frac{4(m-2)^{3}}{a^{3}}\left(4 m^{3}-24 m^{2}+48 m-59\right) .
\end{aligned}
$$

We can verify that the unique real zeros of $\Delta:=Q^{2} / 4-P^{3} / 27$ (which has degree 6 in $m$ ) are $m=2+3(3 / 4 \pm 1 / \sqrt{2})^{1 / 3}$. Hence, if $m \geq 6$, then $\Delta$ has always the same sign, which is clearly positive. When this happens, equation (17) has a unique real solution and two complex conjugate solutions.

If $m=3$, then (17) has the unique real solution

$$
\alpha_{0}=\frac{9+2 \sqrt[3]{2}-3 \sqrt[3]{4}}{31}=0.217 \cdots
$$

Clearly $\alpha_{0}>0$ and $3 \alpha_{0}<1$. Since $\alpha_{1}=2 \alpha_{0}$ we obtain $\alpha_{0}, \alpha_{1}>0$ and $\alpha_{0}+\alpha_{1}<1$. Therefore, $\operatorname{dim}_{H} F=-K\left(\alpha_{0}\right)$. If $m=4$, then (17) has the unique real solution

$$
\alpha_{0}=\frac{9+8 \sqrt[3]{2}-6 \sqrt[3]{4}}{59}=0.161 \cdots
$$

and again $\operatorname{dim}_{H} F=-K\left(\alpha_{0}\right)$. Finally, if $m=5$, then

$$
\operatorname{dim}_{H} F=-K\left(\frac{1+2 \sqrt[3]{2}-\sqrt[3]{4}}{15}\right) .
$$

On the other hand, if $m \geq 6$, then (17) has the unique real solution

$$
\alpha_{0}=\frac{-6 \alpha m^{3}+36 \alpha m^{2}-72 \alpha m+2^{1 / 3} r^{2 / 3}+9 r^{1 / 3}+48 \alpha}{a r^{1 / 3}},
$$

where $\alpha=2^{2 / 3}$ and

$$
\begin{aligned}
r= & 4 m^{6}-48 m^{5}+240 m^{4}-667 m^{3} \\
& +1122 m^{2}-1092 m+\sqrt{(m-2)^{6} a^{2}}+472 .
\end{aligned}
$$


When $r=0$ we must have $m=2$. Therefore, we have $r>0$ for $m \geq 6$. $B y(18), \alpha_{0} \geq 0$ if and only if

$$
s:=-6 \alpha m^{3}+36 \alpha m^{2}-72 \alpha m+2^{1 / 3} r^{2 / 3}+9 r^{1 / 3}+48 \alpha \geq 0 .
$$

It is easy to check that $s=0$ if and only if $m=2$, and that for $m \geq 6$ we have $s \geq 0$. It follows from (18) that $\alpha_{0} \geq 0$. Therefore, $\alpha_{1}=2 \alpha_{0} \geq 0$. Finally, we need to check that $\alpha_{0} \leq 1 / 3$. Clearly,

$$
\alpha_{0}-1 / 3=t /\left(3 a r^{1 / 3}\right),
$$

where

$$
t:=144 \alpha+32 r+32 \cdot 2^{1 / 3} r^{2}-\left(24 m-12 m^{2}+2 m^{3}\right)(9 \alpha+2 r) .
$$

So that $\alpha_{0}-1 / 3 \leq 0$ we must have $t \leq 0$. We can show that $t=0$ if and only if $m=2$, and that $t<0$ for $m \geq 3$. Therefore

$$
\operatorname{dim}_{H} F=-K\left(\frac{-6 \alpha m^{3}+36 \alpha m^{2}-72 \alpha m+2^{1 / 3} r^{2 / 3}+9 r^{1 / 3}+48 \alpha}{a r^{1 / 3}}\right) .
$$

\section{The quadratic case}

We can rewrite $Q(x, y)$ in the form $Q(x, y)=u^{*} A u+v^{*} u+f$, where

$$
u=\left(\begin{array}{l}
x \\
y
\end{array}\right), \quad A=\left(\begin{array}{cc}
a & b / 2 \\
b / 2 & c
\end{array}\right), \quad v=\left(\begin{array}{l}
d \\
e
\end{array}\right) .
$$

Let $\lambda=\sqrt{(a-c)^{2}+b^{2}}$. There exists a rotation matrix $P$ by an angle $\alpha$ such that with the new coordinates $(z, w)^{*}=P^{*} u$ the polynomial $Q$ becomes

$$
\tilde{Q}(z, w)=Q\left(P^{*} u\right)=\lambda_{1} z^{2}+\lambda_{2} w^{2}+\tilde{d} z+\tilde{e} w+f=0,
$$

where

$$
\lambda_{1}=\frac{1}{2}(a+c-\lambda), \quad \lambda_{2}=\frac{1}{2}(a+c+\lambda),
$$

and $\tilde{d}=d \cos \alpha-e \sin \alpha, \tilde{e}=d \sin \alpha+e \cos \alpha$. It follows from (2) that

$$
\operatorname{dim}_{H} F=-\min \left\{R(z, w)+T(z, w) \log _{m} \frac{T(z, w)}{m-2}:(z, w) \in P^{*} \Delta\right\},
$$

where $\Delta=\left\{(x, y) \in[0,1]^{2}: x+y \leq 1\right\}$, and

$$
\begin{aligned}
R(z, w)= & (z \cos \alpha+w \sin \alpha) \log _{m}(z \cos \alpha+w \sin \alpha) \\
& +(-z \sin \alpha+w \cos \alpha) \log _{m}(-z \sin \alpha+w \cos \alpha), \\
T(z, w)= & 1-z(\cos \alpha-\sin \alpha)-w(\sin \alpha+\cos \alpha) .
\end{aligned}
$$


The formula in (19) is too involved to allow determining any "explicit" expression. But at least when $m=3$ there is a simple "geometric" description of $\operatorname{dim}_{H} F$. We first note that each triple $\left(\alpha_{0}, \alpha_{1}, \alpha_{2}\right)$ is in the triangle

$$
\Delta=\left\{(x, y, z) \in[0,1]^{3}: x+y+z=1\right\} .
$$

For simplicity of the exposition we assume in what follows that $\lambda_{1} \lambda_{2}>0$. Then the equation $Q\left(\alpha_{0}, \alpha_{1}\right)=0$ describes an ellipse $E$ in the plane $\alpha_{0} \alpha_{1}$ which intersects the plane defined by $\Delta$ in another ellipse $E^{\prime}$ (for simplicity we shall assume that $E^{\prime}$ is contained in $\Delta$ ). The Hausdorff dimension $\operatorname{dim}_{H} F$ can be described as follows. We consider the function $H: \Delta \rightarrow \mathbb{R}$ given by

$$
H\left(\alpha_{0}, \alpha_{1}, \alpha_{2}\right)=-\sum_{i=0}^{2} \alpha_{i} \log _{3} \alpha_{i} .
$$

Note that its level curves are obtained by solving the equation $x^{x}+y^{y}+z^{z}=$ $c$ for each $c$. We have $\operatorname{dim}_{H} F=H(q)$ where $q$ is any point in the ellipse $E^{\prime}$ that is in the level curve of $H$ closest to the center of $\Delta$. However, it is in general impossible to obtain an explicit analytical expression using this geometric description. In the following sections we obtain some particular formulas when $b=0$.

3.1. The parabolic case. We assume here that $a c=0$ with $(a, c) \neq 0$. We only consider the case $a=0$ and $c \neq 0$ since the other one when $a \neq 0$ and $c=0$ is analogous. We thus have

$$
Q(x, y)=c y^{2}+d x+e y+f .
$$

We make the change $w=y+e /(2 c)$ to obtain the quadratic polynomial

$$
\tilde{Q}(x, w)=c w^{2}+d x+g, \quad g=-\frac{e^{2}}{4 c}+f .
$$

Set

$$
I=[e /(2 c), 1+e /(2 c)], \quad J=[-g,-d-g],
$$

and

$$
\begin{aligned}
& R(w)=\left(w-\frac{e}{2 c}\right) \log \left(w-\frac{e}{2 c}\right)-\frac{c w^{2}+g}{d} \log \frac{c w^{2}+g}{d} \\
& T(w)=1-w+\frac{e}{2 c}+\frac{c w^{2}+g}{d} .
\end{aligned}
$$

We then have the following result. 
Theorem 2. When $d=0$ and $g / c \leq 0$, if $e /(2 c) \in[ \pm \alpha-1, \pm \alpha]$ then setting $\alpha=\sqrt{|g / c|}$,

$$
\begin{aligned}
\operatorname{dim}_{H} F= & -\left( \pm \alpha-\frac{e}{2 c}\right) \log _{m}\left( \pm \alpha-\frac{e}{2 c}\right) \\
& -\left(1 \mp \alpha+\frac{e}{2 c}\right) \log _{m} \frac{1 \mp \alpha+e /(2 c)}{m-1} .
\end{aligned}
$$

When $d \neq 0$ we have

$$
\operatorname{dim}_{H} F=-\frac{1}{\log m} \min \left\{R(w)+T(w) \log \frac{T(w)}{m-2}:\left(w, w^{2}\right) \in I \times J\right\} .
$$

Proof. Note that in the first case we must have $q / c \leq 0$ so that $F \neq \varnothing$, and that by (20) we have $w= \pm \alpha$ with $\alpha=\sqrt{|g / c|}$. This readily implies the first statement (by the general formula in (2)). In the second case we make the change of variables $z=-d x-g$. Then $\tilde{Q}(x, w)=c w^{2}-z$ and the equation $\tilde{Q}(x, w)=0$ describes a parabola. Again the statement follows readily from $(2)$.

3.2. The elliptic case. We now assume that $a c>0$. We make the change of variables $z=x+d /(2 a), w=y+e /(2 c)$ to obtain the quadratic polynomial

$$
\tilde{Q}(z, w)=a z^{2}+c w^{2}-g, \quad g=A^{2}+B^{2}-f,
$$

where $A=d /(2 a)$ and $B=e /(2 c)$. Set

$$
I=[A, 1+A], \quad J=[B, 1+B],
$$

and

$$
\begin{aligned}
& R(z, w)=(z-A) \log _{m}(z-A)+(w-B) \log _{m}(w-B), \\
& T(z, w)=1-z+A-w+B .
\end{aligned}
$$

Proceeding as above we obtain the following result.

Theorem 3. We have

$$
\operatorname{dim}_{H} F=-\min \left\{R(z, w)+T(z, w) \log \frac{T(z, w)}{m-2}:(z, w) \in I \times J\right\} .
$$

We now briefly describe more explicitly the formula in (22).

Case $g=0$. In this case the solution of $\tilde{Q}(z, w)=0$ is $z=w=0$. Then

$$
A \leq 0, \quad B \leq 0, \quad 1+A+B \geq 0
$$

if and only if $F \neq \varnothing$, in which case

$$
\operatorname{dim}_{H} F=A \log _{m}(-A)+B \log _{m}(-B)-(1+A+B) \log _{m} \frac{1+A+B}{m-2} .
$$


Case $g a>0$. Then the equation $\tilde{Q}(z, w)=0$ describes an ellipse and we can write $z=r \cos t, w=s \sin t$, with $t \in[0,2 \pi], r=\sqrt{g / a}$, and $s=\sqrt{g / c}$. If

$$
t \in\left[\min \left\{\cos ^{-1} \frac{A}{r}, \sin ^{-1} \frac{B}{s}\right\}, \max \left\{\cos ^{-1} \frac{1+A}{r}, \sin ^{-1} \frac{1+B}{s}\right\}\right],
$$

then $F \neq \varnothing$ and

$$
\begin{aligned}
\operatorname{dim}_{H} F & =-\min \left\{(r \cos t-A) \log _{m}(r \cos t-A)\right. \\
& +(s \sin t-B) \log _{m}(s \sin t-B) \\
& \left.+(1-r \cos t+A-s \sin t+B) \log _{m} \frac{1-r \cos t+A-s \sin t+B}{m-2}\right\}
\end{aligned}
$$

with the minimum taken over all $t$ as in (23).

3.3. The hyperbolic case $a c<0$. We continue to consider the change of

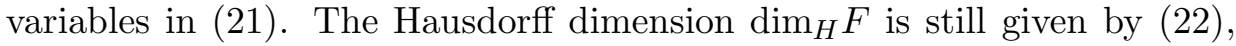
and thus we only describe explicit formulas in each particular case.

Case $g=0$. In this case the solutions of $\tilde{Q}(z, w)=0$ are $z= \pm \sqrt{|c / a|} w$. If $F \neq \varnothing$ then

$$
\begin{aligned}
& \operatorname{dim}_{H} F=-\min \left\{(z-A) \log _{m}(z-A)\right. \\
& \quad+( \pm \sqrt{|a / c|} z-B) \log _{m}( \pm \sqrt{|a / c|} z-B) \\
& \left.\quad+(1-z \mp \sqrt{|a / c|} z+A+B) \log _{m} \frac{1-z \mp \sqrt{|a / c|} z+A+B}{m-2}\right\}
\end{aligned}
$$

with the minimum taken over all $z \in[A, 1+A]$.

Example 6. Let $|a|=|c|$. Consider the functions $K_{+}$and $K_{-}$given by

$$
\begin{aligned}
K_{ \pm}(z)= & (z-A) \log _{m}(z-A)+( \pm z-B) \log _{m}( \pm z-B) \\
& +(1-z \mp z+A+B) \log _{m} \frac{1-z \mp z+A+B}{m-2} .
\end{aligned}
$$

We can easily verify that the unique zero of $K_{-}$is

$$
z=\frac{A-B}{2}, \quad \text { and hence, } \quad w=-z=\frac{B-A}{2} .
$$

We must have $z \geq A,-z \geq B$ and $z+w \leq A+B+1$. These conditions are satisfied if and only if $-1 \leq A+B \leq 0$. In this case $F \neq \varnothing$. We set

$$
D_{-}=(A+B) \log _{m} \frac{-A-B}{2}-(1+A+B) \log _{m} \frac{1+A+B}{m-2} \text {. }
$$


We now consider the function $K_{+}$. The zeros of $K_{+}^{\prime}$ satisfy

$$
\frac{(m-2)^{2}(A-z)(B-z)}{(1+A+B-2 z)^{2}}=1 .
$$

If $m=3$ then

$$
z=\frac{4+3 A+3 B \mp \sqrt{4-3(A-B)^{2}}}{6} .
$$

In order that $z$ is real we must have $-2 / \sqrt{3} \leq A-B \leq 2 / \sqrt{3}$. Furthermore, imposing that $z+w=2 z \leq 1+A+B$ we obtain

$$
\frac{1 \mp \sqrt{4-3(A-B)^{2}}}{6} \leq 0
$$

that is,

$$
z=\frac{4+3 A+3 B-\sqrt{4-3(A-B)^{2}}}{6} \text { with } \sqrt{4-3(A-B)^{2}} \geq 1,
$$

which yields $-1 \leq A-B \leq 1$. It is then easy to check that $z \geq A$ and $z \geq B$, in which case $F \neq \varnothing$. We set $D_{+}=-K_{+}(z)$. If $m=4$ then

$$
z=\frac{1}{4}\left(1+A^{2}+2 A+B^{2}+2 B-2 A B\right) .
$$

Imposing that $z \leq(1+A+B) / 2$ yields $(A-B)^{2} \leq 1$, i.e., $-1 \leq A-B \leq 1$. In this case $z \geq A$ and $w=z \geq B$. Then $F \neq \varnothing$. We set again $D_{+}=$ $-K_{+}(z)$. If $m \geq 5$ then

$$
z=\frac{-4-4 A m-4 B m+A m^{2}+B m^{2} \mp(m-2) \lambda}{2 m(m-4)}
$$

where

$$
\lambda=\sqrt{4-4 A^{2} m+8 A B m-4 B^{2} m+A^{2} m^{2}-2 A B m^{2}+B^{2} m^{2}}
$$

We must have $z \geq A, w=z \geq B$, and $z \leq(1+A+B) / 2$. It follows from the conditions $z \geq A$ and $z \geq B$ that

$$
\frac{-4+4 A m-4 B m-A m^{2}+B m^{2} \mp(m-2) \lambda}{2 m(m-4)} \geq 0
$$

and

$$
\frac{-4-4 A m+4 B m+A m^{2}-B m^{2} \mp(m-2) \lambda}{2 m(m-4)} \geq 0 .
$$

Summing the two inequalities we obtain $-4 \mp(m-2) \lambda \geq 0$, thus yielding

$$
z=\frac{-4-4 A m-4 B m+A m^{2}+B m^{2}+(m-2) \lambda}{2 m(m-4)} \quad \text { and } \quad(m-2) \lambda \geq 4 .
$$


Furthermore, from the condition $z \leq(1+A+B) / 2$ we obtain $\lambda \leq(m-2)$, that is $-1 \leq A-B \leq 1$. It is then easy to check that $(m-2) \lambda \geq 4$ is automatically satisfied. Furthermore, $z \geq A, z \geq B$, and thus $F \neq \varnothing$. We set once more $D_{+}=-K_{+}(z)$. We finally obtain that

$$
\operatorname{dim}_{H} F=\max \left\{D_{-}, D_{+}\right\} .
$$

Case $g \neq 0$. For simplicity of the exposition we only consider the case when $a>0$ and $c<0$. The other case is analogous. Then the equation $\tilde{Q}(z, w)=0$ describes a hyperbola and $z=r \operatorname{ch} t, w=s \operatorname{sh} t$, with $t \in \mathbb{R}$, $r=\sqrt{g / a}$, and $s=\sqrt{g /|c|}$. If

$$
t \in\left[\min \left\{\operatorname{ch}^{-1} \frac{A}{r}, \operatorname{sh}^{-1} \frac{B}{s}\right\}, \max \left\{\operatorname{ch}^{-1} \frac{1+A}{r}, \operatorname{sh}^{-1} \frac{1+B}{s}\right\}\right]
$$

then $F \neq \varnothing$ and

$$
\begin{aligned}
\operatorname{dim}_{H} F= & -\min \left\{(r \operatorname{ch} t-A) \log _{m}(r \operatorname{ch} t-A)\right. \\
& +(s \operatorname{sh} t-B) \log _{m}(s \operatorname{sh} t-B) \\
& \left.+(1-r \operatorname{ch} t+A-s \operatorname{sh} t+B) \log _{m} \frac{1-r \operatorname{ch} t+A-s \operatorname{sh} t+B}{m-2}\right\}
\end{aligned}
$$

with the minimum taken over all $t$ as in (24).

\section{References}

[1] L. Barreira, Hyperbolicity and recurrence in dynamical systems: a survey of recent results, Resenhas IME-USP 5 (2002), 171-230.

[2] L. Barreira, B. Saussol and J. Schmeling, Distribution of frequencies of digits via multifractal analysis, J. Number Theory 97 (2002), 413-442.

[3] A. Besicovitch, On the sum of digits of real numbers represented in the dyadic system, Math. Ann. 110 (1934), 321-330.

[4] P. Billingsley, Ergodic Theory and Information, Wiley and Sons, 1965.

[5] H. Eggleston, The fractional dimension of a set defined by decimal properties, Quart. J. Math. Oxford Ser. 20 (1949), 31-36. 